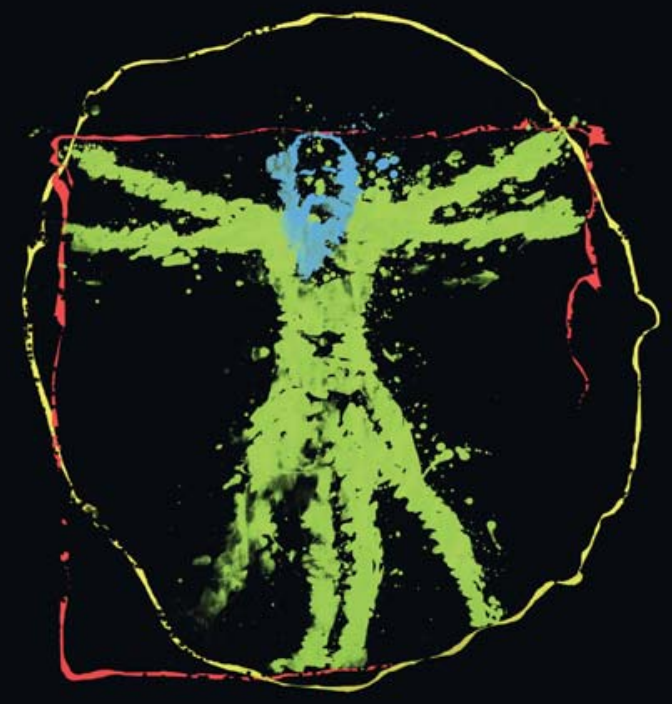

Susanne Hartwig (Hg.)

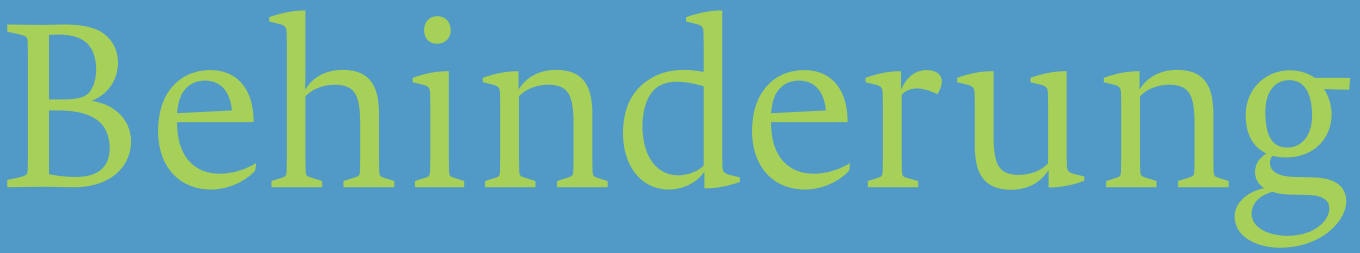

Kulturwissenschaftliches Handbuch 
2002 J.B.METZLER 
Susanne Hartwig (Hg.)

Behinderung:

Kulturwissenschaftliches Handbuch 


\section{Die Herausgeberin}

Susanne Hartwig ist Professorin für Romanische Literaturund Kulturwissenschaft an der Universität Passau.

ISBN 978-3-476-05737-2

ISBN 978-3-476-05738-9 (eBook)

https://doi.org/10.1007/978-3-476-05738-9

Die Deutsche Nationalbibliothek verzeichnet diese Publikation in der Deutschen Nationalbibliografie; detaillierte bibliografische Daten sind im Internet über http://dnb.d-nb.de abrufbar.

\section{J. B. Metzler}

(C) Springer-Verlag GmbH Deutschland, ein Teil von Springer Nature, 2020

Das Werk einschließlich aller seiner Teile ist urheberrechtlich geschützt. Jede Verwertung, die nicht ausdrücklich vom Urheberrechtsgesetz zugelassen ist, bedarf der vorherigen Zustimmung des Verlags. Das gilt insbesondere für Vervielfältigungen, Bearbeitungen, Übersetzungen, Mikroverfilmungen und die Einspeicherung und Verarbeitung in elektronischen Systemen.

Die Wiedergabe von allgemein beschreibenden Bezeichnungen, Marken, Unternehmensnamen etc. in diesem Werk bedeutet nicht, dass diese frei durch jedermann benutzt werden dürfen. Die Berechtigung zur Benutzung unterliegt, auch ohne gesonderten Hinweis hierzu, den Regeln des Markenrechts. Die Rechte des jeweiligen Zeicheninhabers sind zu beachten.
Der Verlag, die Autoren und die Herausgeber gehen davon aus, dass die Angaben und Informationen in diesem Werk zum Zeitpunkt der Veröffentlichung vollständig und korrekt sind. Weder der Verlag, noch die Autoren oder die Herausgeber übernehmen, ausdrücklich oder implizit, Gewähr für den Inhalt des Werkes, etwaige Fehler oder Äußerungen. Der Verlag bleibt im Hinblick auf geografische Zuordnungen und Gebietsbezeichnungen in veröffentlichten Karten und Institutionsadressen neutral.

Umschlagabbildung: (C) dieKleinert / Thure Röttger / mauritius images

J. B. Metzler ist ein Imprint der eingetragenen Gesellschaft Springer-Verlag GmbH, DE und ist ein Teil von Springer Nature

Die Anschrift der Gesellschaft ist:

Heidelberger Platz 3, 14197 Berlin, Germany 


\section{A Definitionen und Konzepte}

\section{Definitionen und Klassifikationen}

Akteur*innen und Disziplinen bzw. Professionen haben - je nach Handlungskontext - Konstrukte von Behinderung entwickelt, mit denen unterschiedliche Interessen verfolgt werden. Anhand unterschiedlicher Modelle, Klassifikationen und Gesetzesnormen wird in diesem Beitrag analysiert, wie Behinderung und Nichtbehinderung konzeptualisiert werden. Dies wird exemplarisch erörtert: an der aktuellen Behinderungsklassifikation der Weltgesundheitsorganisation, der Behinderungs-Definition des Sozialgesetzbuches IX in der Neufassung des Bundesteilhabegesetzes (BTHG) der Bestimmung von Behinderung in der UN-Behindertenrechtskonvention (UN-BRK) als einfachem deutschen Recht sowie an den Definitionen, die im Sozialen Modell der britischen Disability Studies bedeutsam sind. Abschließend wird die Bedeutung von Definitionen und Klassifikationen reflektiert und kurz resümiert.

\subsection{Wie und wozu Konstruktionen?}

Gesellschaftliche Phänomene existieren nur durch ihre und in ihrer gesellschaftlichen Konstruktion. Etwas ,Natürliches، besteht nicht an sich, sondern immer nur in Beobachtung durch Menschen oder durch von Menschen konstruierte Maschinen (wie Kameras, Drohnen u. a.).

Traditionell wird klassifiziert, was im öffentlichen oder im Fachdiskurs als ungewöhnlich oder auch problematisch beurteilt wird. Dies wird als besonderes Phänomen zum Forschungsgegenstand gemacht. Es sind zumeist Phänomene, die nicht dem herrschenden Maßstab, dem formulierten oder unbewusst verfolgten Ideal entsprechen. Seit Jahrhunderten sind dies körperliche, seelische und geistige Phänomene, je nach Ort und Zeit wurde Unterschiedliches hierzu gezählt. Mit der Entstehung internationaler Fachgesellschaften im 19. Jahrhundert und deren Interesse an international gültigen Regelwerken hat sich der Fokus auf Störungen und Abweichungen von dem als normal oder gewöhnlich konstruierten Phänomen verschärft (vgl. Hirschberg 2009, 32-61). Während in früheren Jahrhunderten regional große Unterschiede vorherrschten, wie mit körperlichen, seelischen und geistigen Phänomenen, die auch als Beeinträchtigungen verstanden wurden, umgegangen wurde, hat sich seit der Neuzeit die Orientierung am wirtschaftlich funktions- und leistungsfähigen, verwertbaren Körper verstärkt (vgl. Foucault 1968, 104; Dörner 2017, 692-700). Mit dieser Orientierung verbunden ist die angestrebte internationale Vereinheitlichung von Diagnosen und Klassifizierungsordnungen.

Nach dem Zweiten Weltkrieg wurde die Weltgesundheitsorganisation (WHO) als Folgeorganisation des internationalen statistischen Kongresses gegründet und hat dessen Entwicklung internationaler Klassifikationen von Krankheiten (ab 1980 von Behinderungen) übernommen (vgl. Hirschberg 2009, 35). Stetig wurden diese Diagnosekataloge ausgeweitet bzw. gesellschaftlichen Vorstellungen angepasst, wie besonders an der Klassifizierung von Homosexualität als Störung im Diagnostic Statistical Manual II (DSM-II) und deren späterer Aufhebung im DSM-IV deutlich wird.

Die Macht, Normalität und Abweichung zu determinieren, verweist auf das darunterliegende Bedürfnis, Ordnungen herzustellen oder zu sichern, das speziell vor dem Hintergrund der leistungsorientierten gesellschaftlichen Matrix zu begreifen ist. Konstruktionen von Abweichungen sind als Störung oder Beeinträchtigung grundsätzlich räumlich und zeitlich verortet; universale, örtlich und räumlich unabhängige Störungen oder Beeinträchtigungen gibt es nicht. Sie haben, wie Foucault für Geisteskrankheiten analysiert, »ihre Wirklichkeit und ihren Wert als Krankheit nur innerhalb einer Kultur, die sie als solche erkennt « (Foucault 1968, 93).

Kurz zusammengefasst gibt es Phänomene, die als Störung oder Beeinträchtigungbezeichnet, diagnosti- 
ziert und klassifiziert werden. Diese Beurteilungen werden von medizinischen oder auch psychiatrischen Fachpersonen vorgenommen und können somit nur als zu einem bestimmten Zeitpunkt (selbst unter dem Anspruch der internationalen Gültigkeit) und mit räumlichem Bezug geltende Perspektive erachtet werden. Auch wenn es sich immer um ein Konstrukt und nicht um einen natürlichen Gegenstand handelt, sind diese Konstruktionen von Störungen oder Abweichungen aufgrund ihres verbreiteten Einsatzes in den Feldern Medizin, Psychiatrie, Rechtswesen, Pädagogik und allen humanwissenschaftlichen Disziplinen machtvoll.

\subsection{Konstruktion von Behinderung als Abweichung}

Nicht nur die Weltgesundheitsorganisation, auch andere Akteur*innen haben Behinderung konstruiert und machtvolle Definitionen erstellt. Sie versuchen, diese Definitionen zu verbreiten, ihre Perspektive auf Behinderung wirkmächtig werden zu lassen. Hierbei ist jedoch zu unterscheiden, ob die Autor*innen von Behinderungskonstruktionen gesellschaftlich anerkannte Akteur*innen sind oder nicht. Seit der Neuzeit sind die Disziplinen Medizin, Psychiatrie (und ihre Nachbarwissenschaften) vorherrschend in der Konstruktion des Abweichenden: Krankheit als Abweichung von Gesundheit oder Behinderung als Abweichung von Funktionsfähigkeit. Die Durchsetzung der Interessen gesellschaftlicher Akteur*innen lässt sich auch daran erkennen, dass Behinderung bis 1980 mit der Krankheitsklassifikation (ICD) beurteilt wurde. Erst seit 1980 wird Behinderung eigenständig klassifiziert und nicht mehr unter den Krankheitsbegriff subsumiert (vgl. WHO 1980; Hirschberg 2009, 46-57).

Durch Klassifikationen spricht die anerkannte Profession, die in der historischen Abfolge der Entwicklung von Klassifikationsinstrumenten wiederkehrend hierzu beauftragt ist, vor dem Hintergrund der gesellschaftlich dominanten Diskurse und der herrschenden Verhältnisse. Dies waren mehrheitlich die Professionen der Medizin und der Psychiatrie - im Anschluss an die Entwicklung der botanischen Klassifikationen (Carl von Linné), aufbauend auf den medizinischen Systematisierungen durch Thomas Sydenham im 17. Jahrhundert, deren Einfluss im Rahmen der Perspektive der (ehemaligen imperialistischen) dem Völkerbund angehörigen Staaten zu verorten ist (vgl. Hirschberg 2009,28-38).
Klaus Dörner veranschaulicht die Entstehungsgeschichte von Psychiatrien auch an der Unterscheidung, wer nicht als arbeitsfähig und vernünftig galt (vgl. Dörner 2017, 692-695). Wer spricht, wer entscheidet, was wie klassifiziert wird? Dies sind diejenigen, die Positionen erworben haben, in denen ihnen die Entscheidungsmacht zugesprochen wird, Syndrome oder Phänomene als behindert oder nichtbehindert zu klassifizieren.

Gesellschaftliche Konstruktionen sind mit dem Zugang zu Ressourcen verbunden (vgl. Gregory 1997). Die jeweils als bedeutsam anerkannte Konstruktion funktioniert somit als Instrument, das zur Durchsetzung von Interessen und gesellschaftlichen Ordnungen genutzt werden kann. Die konstruierten Definitionen werden als Instrument gebraucht, um mit ihnen unterschiedliche Interessen zu verfolgen. Sie bieten einen Einblick sinto the nature of the 'powers-that-ber who make and then use definitions to build, maintain, and advance their position in society“ (Gregory 1997, 487). Definitionen können dazu genutzt werden, die gesellschaftliche Position und den eigenen Einflussbereich aufzubauen, aufrecht zu erhalten oder sogar zu erweitern. Hierbei ist entscheidend, was definiert wird und was nicht.

Das traditionell herrschende Modell konstruiert Behinderung aus medizinischer, defizitorientierter Perspektive. Diese Perspektive lässt sich auf die seit der Neuzeit vorherrschenden gesellschaftlichen Interessen an einer produktivitätsorientierten Verwertung der Körper zurückführen. Sie ist auch durch eine ontologische Betrachtung auf Körper begründet, diese auf ihr Sosein und durch die Produktivitätsorientierung auf ihre Leistungsfähigkeit zu reduzieren.

Mit der auf die Ausbeutung der Arbeitskraft fokussierten Industrialisierung wurde die Aufgabe von Medizin und Psychiatrie umso differenzierter, als Kriterien für die Arbeitsfähigkeit bei Krankheiten wie beispielsweise die Festlegung der Normaltemperatur entwickelt wurden (vgl. Hess 1999, 224; zur Analyse des ärztlichen Blicks in den entstandenen Kliniken Foucault 1973) (s. Kap. 42). Machtanalytisch gefasst wurden die Körper normiert, abweichende Entwicklungen sanktioniert und die Ausführungs- und Leistungsfähigkeit überprüft, um dann passend zur bestmöglichen Ausnutzung der individuellen Fähigkeiten zur Arbeit eingesetzt zu werden (vgl. Foucault 1994, 241-248).

Festzuhalten ist, dass das medizinische, auf das Individuum ausgerichtete Modell von Behinderung nicht nur im Kontext von Medizin und Psychiatrie. 
sondern auch im Rahmen der wirtschaftlichen Interessen seit der Neuzeit reflektiert werden muss (s. Kap. 4). Durch die Bismarckschen Sozialgesetze Ende des 19. Jahrhunderts, die Kranken-, Unfall, Renten- und Angestellten-/Arbeitslosenversicherung, wurde der Erhalt oder die Wiederherstellung der Leistungs- und Arbeitsfähigkeit nicht nur angestrebt, sondern auch (wenn auch nicht umfassend) abgesichert. Mit diesen Errungenschaften des Wohlfahrtsstaates wurde die definierte, konstruierte Feststellung einer Behinderung mit einem individuellen Leistungsanspruch verbunden. Eine Behinderung gilt als »naturbedingter Nachteil«, aufgrund dessen die jeweilige Person einen Nachteilsausgleich erhält (vgl. Hirschberg 2009, 263-267; in ihrer Bedeutung für Rehabilitation und Teilhabe Welti 2005).

Die gesellschaftlichen Partizipationschancen sind stark an die Erwerbstätigkeit eines Menschen gekoppelt (vgl. aus intersektionaler Perspektive LibudaKöster/Schildmann 2016). Auch das gesellschaftliche Bewusstsein ist an Nützlichkeit und Leistungsfähigkeit orientiert, und weniger an einer wahrgenommenen Bandbreite von Nicht_behinderung oder an den gesellschaftlichen Benachteiligungsstrukturen, die im sozialen Modell von Behinderung fokussiert werden (s. u.).

\subsection{Klassifikationen, Modelle, Gesetzes- normen: Unterschiedliche Perspektiven auf Behinderung}

Mit der humanwissenschaftlichen Konstruktion von Behinderung als Forschungsgegenstand in Medizin, Psychologie, Pädagogik und weiteren Disziplinen wird auf den Hilfebedarf, die Abhängigkeit von anderen, die Linderung oder auch Verhinderung von Beeinträchtigung fokussiert. Behinderte Menschen sollen in ihren Fähigkeiten gefördert und an die nichtbehinderte Gesellschaft angepasst werden. Aus dieser Perspektive sind medizinische Klassifikationen und juristische Konstruktionen konstruiert. Sie stellen Behinderung in den Vordergrund, Nichtbehinderung wird entweder nicht oder nur indirekt thematisiert.

Aufgrund des Bestrebens von Rehabilitationsfachkräften und anderen Akteur*innen, Krankheit und Behinderung zu unterscheiden, hat die Weltgesundheitsorganisation in den 1970er Jahren die erste Klassifikation von Behinderung entwickelt, die »International Classification of Impairment, Disability, and Handicap«(ICIDH).Diese differenzierte in Bezug auf
- die körperliche Ursache (oder Folge einer Krankheit): impairment

- die individuellen Folgen für die eigene Handlungsfähigkeit: disability

- die Auswirkungen hinsichtlich der gesellschaftlichen Rollenperformanz: handicap.

Als zu linear und kausal kritisiert, wurde die Behinderungsklassifikation von einer Vielzahl von Akteur*innen und daraufhin von verschiedenen $\mathrm{Ge}$ sundheitsfachleuten weiterentwickelt, wobei ein interaktives Verhältnis mehrerer Komponenten den früheren kausalen Ansatz ersetzen sollte. Die zweite Behinderungsklassifikation, die »International Classification of Functioning, Disability, and Health « (ICF) wurde von der Vollversammlung der Weltgesundheitsorganisation 2001 verabschiedet.

In der Weiterentwicklung von der ersten zur zweiten Behinderungsklassifikation zeigt sich, dass mit dieser nicht nur Behinderung definiert, kategorisiert und klassifiziert wird, sondern Behinderung auch von einem Pendant, Funktionsfähigkeit, begleitet wird. Der dritte Oberbegriff, Gesundheit, bildet allerdings keinen beide umspannenden Rahmen, sondern verdoppelt in gewisser Weise die Bedeutung von Funktionsfähigkeit als Orientierung, als das Eine, von dem Behinderung als abweichend gemessen wird (vgl. Hirschberg 2009, 202-207).

Definiert wird Behinderung in der ICF in zweierlei Hinsichten. Behinderung bildet erstens den Oberbegriff von drei Komponenten und wird zweitens als Ergebnis des Wechselverhältnisses mehrerer Komponenten gefasst:

"Disability is an umbrella term for impairments, activity limitations and participation restrictions. It denotes the negative aspects of the interaction between an individual (with a health condition) and that individual's contextual factors (environmental and personal factors)." (WHO 2001, 213)

Die Definition von Funktionsfähigkeit ist parallel zu der von Behinderung mit positiven Komponenten konstruiert, jedoch werden nur die Komponenten von Behinderung klassifiziert. Funktionsfähigkeit fungiert gleichermaßen als Normalitätswert, als Maßstab für Behinderung, von dem diese (in ihren unterschiedlichen Komponenten) als graduelle Abweichung beurteilt wird (vgl. Hirschberg 2009, 240-243). Mit der neuen Komponente der Kontextfaktoren, in der besonders die Umweltfaktoren (differenziert in Barrieren oder_Unterstïtzungsfaktoren)_hervorzuheben_sind, 
wird zwar die gesellschaftliche Dimension von Behinderung betont - dies verändert jedoch nicht die generelle Konstruktion von Behinderung als Abweichung von Funktionsfähigkeit bzw. Nichtbehinderung.

Insgesamt ist diskursanalytisch herauszustellen, dass Behinderung und Normalität in der ICF zwar variabel und flexibel konstruiert sind, jedoch auf einer protonormalistischen Grundlage, die in der Gegenüberstellung und der einseitigen negativen Beurteilung von Behinderung begründet ist (vgl. Hirschberg 2009, 299-308; zur Normalismustheorie Link 2009). Aus der Diskursanalyse lässt sich zudem folgern, wie Klassifikationen als gesellschaftlich geronnene Diskurse und Praktiken Behinderung produzieren und konstruieren: doing disability.

Mit der Verabschiedung des Bundesteilhabegesetzes im Dezember 2016 wird das Sozialgesetzbuch IX mit dem Titel »Rehabilitation und Teilhabe behinderter Menschen« (2001) reformiert, es tritt in Stufen bis 2020 in Kraft. Es fasst Behinderung in $\$ 2$ SGB IX geltend seit dem 01.01.2018 - wie folgt:

"(1) Menschen mit Behinderungen sind Menschen, die körperliche, seelische, geistige oder Sinnesbeeinträchtigungen haben, die sie in Wechselwirkung mit einstellungs- und umweltbedingten Barrieren an der gleichberechtigten Teilhabe an der Gesellschaft mit hoher Wahrscheinlichkeit länger als sechs Monate hindern können. (2) Eine Beeinträchtigung nach Satz 1 liegt vor, wenn der Körper- und Gesundheitszustand von dem für das Lebensalter typischen Zustand abweicht. (3) Menschen sind von Behinderung bedroht, wenn eine Beeinträchtigung nach Satz 1 zu erwarten ist."

Auch die Neufassung des SGB IX von 2018 charakterisiert Behinderung als Abweichung der individuellen Funktion, Fähigkeit oder Gesundheit vom für das Lebensalter eines Menschen typischen, als normal angesehenen Zustand. Sie nutzt weitere Kriterien: die Alterstypik sowie die hohe Wahrscheinlichkeit, mit der eine Beeinträchtigung länger als ein halbes Jahr von der für das jeweilige Lebensalter erwarteten Typik abweicht. Diese Normalitätsorientierung - gekennzeichnet durch die drei Merkmale der Wahrscheinlichkeit, der Abweichung und der Alterstypik - grenzt die Bestimmung von Behinderung entscheidend ein.

Somit orientiert sich diese Behinderungsdefinition, trotz des Aufgreifens der Wechselwirkung von Beeinträchtigungen mit Barrieren, noch stark an einem individualistischen Verständnis von Behinderung. Das
Kriterium der Dauer von sechs Monaten bietet nicht mehr als einen Anhaltspunkt, da chronische Erkrankungen aufgrund intensiverer oder schwächerer Schübe unterschiedlich lange dauern können. Chronische Erkrankungen lassen sich daher über das Kriterium der Dauer nicht adäquat erfassen. Das Alterskriterium erscheint nur plausibel für jüngere Altersgruppen, für Menschen höheren Alters nicht. Ältere Menschen sind häufig gebrechlich und pflegebedürftig; in dieser Altersgruppe ist es dementsprechend sogar typisch, beeinträchtigt zu sein. Die Beschreibung des Alters verweist daher eher auf die Abgrenzung zwischen Eingliederungshilfe und Pflege, also auf unterschiedliche finanzielle Budgets und deren Begründungen.

Die seit 2018 anteilig an der BRK ausgerichtete Definition ist leitend für die Bewilligung von Leistungen für behinderte Menschen. Wie das gesamte deutsche Recht soll auch das SGB IX (BTHG) und hiermit auch diese Definition im Licht der am 26. März 2009 in Deutschland als einfaches Recht in Kraft getretenen UN-BRK gelesen werden. Inwiefern dies vollzogen wird, ist an der Rechtsprechung nicht einheitlich $\mathrm{zu}$ erkennen, sondern vielschichtig (vgl. http://www. reha-recht.de/ Aufruf vom 9. Dezember 2019).

\subsection{Konvention über die Rechte von Menschen mit Behinderungen}

Auch wenn die vorher verabschiedeten Menschenrechtsverträge für alle Menschen und folglich auch für behinderte Menschen gelten, wurde beschlossen, die Rechte behinderter Menschen noch einmal explizit herauszustellen und deren Rechtsausübungsmöglichkeiten zu stärken (vgl. Degener 2009). Die UN-BRK ist eine Antwort auf die systematische und strukturelle Benachteiligung behinderter Menschen weltweit.

Mit der UN-BRK sind die Staaten, die die Konvention unterzeichnet und ratifiziert haben, verpflichtet, das Ziel zu verfolgen, »den vollen und gleichberechtigten Genuss aller Menschenrechte und Grundfreiheiten durch alle Menschen mit Behinderungen $\mathrm{zu}$ fördern, zu schützen und zu gewährleisten und die Achtung der ihnen innewohnenden Würde zu fördern«(Art. 1 Abs. 1 BRK). Definiert wird nicht Behinderung, sondern die Gruppe behinderter Menschen:

"Zu den Menschen mit Behinderungen zählen Menschen, die langfristige körperliche, seelische, geistige oder Sinnesbeeinträchtigungen haben, welche sie in Wechselwirkung mit verschiedenen Barrieren an der 
vollen, wirksamen und gleichberechtigten Teilhabe an der Gesellschaft hindern können." (BRK, Art. 1 Abs. 2; vgl. Hirschberg 2011)

Im Gegensatz zur Definition des SGB IX (BTHG 2018) wird Behinderung hier - ähnlich wie in der ICF - als Ergebnis der Wechselwirkung von Beeinträchtigungen und Barrieren konstruiert. Diese Definition ist hinsichtlich des zeitlichen Kriteriums offener als die SGB IX-Definition (in der BTHG-Fassung).

In der UN-BRK wird herausgestellt, dass Barrieren die Teilhabe behinderter Menschen einschränken. Jeder Vertragsstaat muss behinderten Menschen den Zugang zu allen Lebensbereichen ermöglichen, damit sie ihre Rechte ausführen und genießen können. Mit dem häufig verwendeten Begriff sgleichberechtigt، wird verdeutlicht, dass behinderte Menschen aus Menschenrechtsperspektive gleichberechtigt mit nichtbehinderten Menschen sind.

\subsection{Die Konstruktion von Behinderung aus Perspektive der Disability Studies}

Die Kontroverse zwischen medizinischem und sozialem Modell von Behinderung lässt sich auch als Kampf um die Definitionsmacht bezeichnen, ob Behinderung als durch individuelle Beeinträchtigungen oder gesellschaftliche Benachteiligung verursacht wird. Das soziale Modell von Behinderung wurde von der britischen Behindertenbewegung seit den 1970er Jahren entwickelt, ähnlich auch das US-amerikanische Minderheitenmodell (vgl. Hirschberg/Köbsell 2016). Der Fokus liegt auf gesellschaftlichen Barrieren und Ausschlussprozessen, durch die beeinträchtigte Menschen von der nichtbehinderten Mehrheitsgesellschaft benachteiligt werden (s. u.).

Michael Oliver, einer der britischen Begründer der Disability Studies, fasst zusammen, wie Behinderung in den letzten 100 Jahren konstruiert wurde: zum einen als medizinisches Problem, das möglichst geheilt oder gelindert, und zum zweiten als soziales Problem, das sozial versorgt werden soll:

"In the past 100 years or so, industrial societies have produced disability first as a medical problem requiring medical intervention and second as a social problem requiring social provision. Research, on the whole, has operated within these frameworks and sought to classify, clarify, map and measure their dimensions" (Oliver 1992 101)
In den britischen Disability Studies wurden Beeinträchtigung und Behinderung in den Prinzipien der Union of the Physically Impaired Against Segregation (UPIAS) definiert, und zwar: impairment als »lacking all or part of a limb, or having a defective limb, organ or mechanism of the body « und disability als

"[...] the disadvantage or restriction of activity caused by a contemporary social organisation which takes no or little account of people who have physical impairments and thus excludes them from participation in the mainstream of social activities." (UPIAS 1975)

Diese grundlegende Definition analysiert die gesellschaftlichen Verhältnisse in ihrer Wirkung für beeinträchtigte Menschen, die gesellschaftlich benachteiligt und behindert werden.

\subsection{Fazit}

Die Analyse der machtvollen Konstruktion von Behinderung und Nichtbehinderung in den anerkannten sozialrechtlichen Konstruktionen und Klassifikationen ebenso wie in der UPIAS-Definition von Behinderung zeigt, dass Behinderung in Abgrenzung zu Nichtbehinderung, auch als Funktionsfähigkeit bezeichnet, konzeptualisiert wird.

Ebenfalls wird deutlich, dass Gesellschaften von Aushandlungspraxen geprägt sind, was besonders den Zugang zu gesellschaftlichen Ressourcen bzw. die Erlangung von Chancen betrifft. Definitionen und Klassifikationen haben keinen Selbstzweck, sondern werden in Aushandlungsprozessen mit unterschiedlichen Zielen eingesetzt. Aus Perspektive der Disability Studies ist es entscheidend, diese unterschiedlichen Interessen und Praxen transparent zu machen und Definitionen und Klassifikationen für das Ziel gleichberechtigter gesellschaftlicher Teilhabe aller behinderten Menschen einzusetzen.

\section{Literatur}

Degener, Theresia: Menschenrechte und Behinderung. In: Markus Dederich/Wolfgang Jantzen (Hg.): Behinderung und Anerkennung. Behinderung, Bildung, Partizipation. Enzyklopädisches Handbuch der Behindertenpädagogik. Stuttgart 2009, 160-169.

Dörner, Klaus: Wege der Psychiatrie (Psychiatriegeschichte). In: Klaus Dörner/Ursula Plog/Thomas Bock/Peter Brieger/Andreas Heinz/Frank Wendt (Hg.): Irren ist menschlich. Lehrbuch der Psychiatrie und Psychotherapie. Köln 2017, 687-714 
Foucault, Michel: Psychologie und Geisteskrankheit. Frankfurt a. M. 1968.

Foucault, Michel: Die Geburt der Klinik. Eine Archäologie des ärztlichen Blicks. München 1973.

Foucault, Michel: Überwachen und Strafen. Die Geburt des Gefängnisses. Frankfurt a. M. 1994.

Gregory, Robert: Definitions as Power. In: Disability and Rehabilitation 19/11 (1997), 487-489.

Hess, Volker: Die moralische Ökonomie der Normalisierung. Das Beispiel Fiebermessen. In: Werner Sohn/Herbert Mehrtens (Hg.): Normalität und Abweichung. Studien zur Theorie und Geschichte der Normalisierungsgesellschaft. Opladen 1999, 222-243.

Hirschberg, Marianne: Behinderung im internationalen Diskurs. Die flexible Klassifikation der Weltgesundheitsorganisation. Frankfurt a. M. 2009.

Hirschberg, Marianne: Behinderung: Neues Verständnis nach der Behindertenrechtskonvention (Positionen der Monitoring-Stelle, Nr. 4). Berlin 2011.

Hirschberg, Marianne/Köbsell, Swantje: Grundbegriffe und Grundlagen: Disability Studies, Diversity und Inklusion. In: Ingeborg Hedderich/Gottfried Biewer/Judith Hollenweger/Reiner Markowetz (Hg.): Handbuch Inklusion und Sonderpädagogik. Bad Heilbrunn 2016, 555-568.
Libuda-Köster, Astrid/Schildmann, Ulrike: Institutionelle Übergänge im Erwachsenenalter (18-64 Jahre). Eine statistische Analyse der Verhältnisse zwischen Behinderung und Geschlecht. In: Vierteljahresschrift für Heilpädagogik und ihre Nachbargebiete 85/1 (2016), 7-24.

Link, Jürgen: Versuch über den Normalismus. Wie Normalität produziert wird. Wiesbaden ${ }^{3} 2009$.

Oliver, Michael: Changing the Social Relations of Research Production? In: Disability, Handicap \& Society 7/2 (1992), 101-114.

Union of the Physically Impaired Against Segregation (UPIAS): Fundamental principles of Disability. London 1975.

Welti, Felix: Behinderung und Rehabilitation im sozialen Rechtsstaat - Freiheit, Gleichheit und Teilhabe behinderter Menschen. Tübingen 2005.

WHO/World Health Organisation: The International Classification of Impairments, Disabilities and Handicaps. Genf 1980.

World Health Organisation: The International Classification of Functioning, Disability and Health. Genf 2001.

Marianne Hirschberg 